Memorias del VII Encuentro Nacional de Experiencias en la Enseñanza de la Biología y la Educación Ambiental y II Congreso Nacional de Investigación en la Enseñanza de la Biología

\title{
MENOS RESIDUOS ORGÁNICOS A LA BASURA, MÁS COMPOSTAJE POR LOMBRICULTIVO EN LA UNIVERSIDAD PEDAGÓGICA NACIONAL. UNA ESTRATEGIA PARA LA SUSTENTABILIDAD DESDE EL AULA.
}

\section{ORGANIC WASTE UNLESS IT OUT, MORE VERMICULTURE COMPOSTING BY NATIONAL TEACHING AT THE UNIVERSITY. A STRATEGY FOR SUSTAINABILITY FROM THE CLASSROOM.}

\author{
Piñeros, Johana ${ }^{1}$, \\ Vargas, Juan Camilo², \\ Franco, Ricardo. $\mathrm{A}^{3}$
}

\section{Resumen}

Esta ponencia consiste en la formulación de una estrategia de saneamiento ambiental para tratar la problemática alrededor del manejo de residuos sólidos orgánicos generados en el Departamento de Química de la Facultad de Ciencia y Tecnología de la Universidad Pedagógica Nacional; se diseñarán herramientas que contribuyan a la mitigación de la contaminación por residuos desde el compostaje producido desde la lombricultura, a partir de su aplicación y utilización por medio de la separación y reutilización de residuos sólidos, dirigido hacia la enculturación entorno al correcto manejo de residuos según las normas vigentes. Se focalizará en la población estudiantil de los espacios académicos de Educación Ambiental y Sistemas Orgánicos II, para promover por medio de metodologías la interacción de la comunidad educativa para mejorar la gestión integral de los Residuos sólidos orgánicos.

\section{Abstract}

This paper consists in the making of environmental sanitation strategy to address issues around the management of organic solid waste generated in the Department of Chemistry, Faculty of Science and Technology of the National Pedagogical University. Will be design tools that contribute to the mitigation of residue contamination by the compost produced from the vermiculture, from the application and use by separation and reuse of solid waste, directed toward the enculturation around the correct waste management according to current regulations. Will be focused on the student population in academic spaces Environmental Education and Organic Systems II, to promote methodologies

\footnotetext{
${ }^{1}$ Estudiante Licenciatura en Química, Universidad Pedagógica Nacional. Bogotá, Colombia. Monitora de Investigación. Fracyjohana911@hotmail.com

${ }^{2}$ Estudiante Licenciatura en Química, Universidad Pedagógica Nacional. Bogotá, Colombia. chemisjcv@gmail.com

${ }^{3}$ Profesor Departamento de Química, Universidad Pedagógica Nacional. Bogotá, Colombia. Grupo IREC
} 
Bio-grafia Escritos sobre La Biología y su Enseñanza.

Edición Extra-Ordinaria. ISSN 2027-1034 P. p113 - 121

Memorias del VII Encuentro Nacional de Experiencias en la Enseñanza de la

Biología y la Educación Ambiental y II Congreso Nacional de Investigación en la Enseñanza de la Biología

through the interaction of the educational community improving the integrated management of organic solid waste.

Palabras Clave Compostaje, Residuos biodegradables, separación en la fuente, lombricultivo, sustentabilidad.

Keyword Composting, biodegradable waste, source separation, vermiculture, sustainability.

\section{Introducción}

Se conoce que la lombricultura es un proceso limpio y de fácil aplicación en el momento de reciclar, que por medio del compostaje optimiza el proceso natural por el que los restos orgánicos retornan al suelo cerrando el ciclo de la materia y disminuyendo el tiempo para obtener el producto final. La lombricultura está presente en diversas actividades que pasan de ser hobby, hasta ser un factor comercial, inclusive en la industria farmacéutica tiene una gran aplicación, como en antibióticos o suplementos de dietas. (Schuldt, 2006). La tecnología de tratamiento para reciclaje de residuos orgánicos y producción de abonos y proteína, gestiona su desarrollo en la cría intensiva de lombrices, que por medio de la descomposición natural generada en su complejo sistema digestivo recicla materia orgánica (Barbado, 2004); en este caso se centra el interés en la lombriz roja californiana denominada también Red hybrid, de donde se derivan especies como: Eisenia fetida, Eudrilus Eugeniae, Dendrobaena veneta o Perionyx excavatus (Padilla y Cuesta, 2003).

\section{Recolección del alimento}

Las lombrices en general pueden descomponer diversos alimentos y sustancias, la lombriz roja californiana de igual forma lo hace, pero requiere que la materia orgánica suministrada como alimento este parcial o totalmente descompuesta, de lo contrario la elevada temperatura de hasta $75^{\circ} \mathrm{C}$ generada por la fermentación matara a las lombrices (Rodríguez, 2005).

\section{Hábitat}

Es importante tener en cuenta ciertos aspectos en relación a la forma de vida de las lombrices, esta especie habita los primeros $50 \mathrm{~cm}$ del suelo, lo cual implica susceptibilidad al clima. Los rayos ultravioletas también las perjudican considerablemente (fotofóbica), al igual que una excesiva humedad (70\%), Temperatura $\left(12-25^{\circ} \mathrm{C}\right)$ acidez del medio $(\mathrm{pH} 7)$ e incorrecta alimentación. (Rodríguez, 2005) 
Bio-grafía Escritos sobre la Biología y su Enseñanza.

Edición Extra-Ordinaria. ISSN 2027-1034 P. p113 - 121

Memorias del VII Encuentro Nacional de Experiencias en la Enseñanza de la

Biología y la Educación Ambiental y II Congreso Nacional de Investigación en la Enseñanza de la Biología

\section{Aspectos claves en el lombricultivo}

En referencia a la población a tratar con la estrategia, se considera la que la explotación será de tipo mediano, empleando áreas considerables y con disponibilidad confiable de recursos, las dimensiones mínimas para la construcción de los cajones para las camas o lechos de las lombrices es de $40 \times 70 \times 15 \mathrm{~cm}$, el material empleado puede ser desde tablas de madera, ladrillo, geomembranas o por simples camas en motón (García, 2005).Los cajones deben ser construidos con tapa y base perforada para permitir la aireación del sustrato y el drenaje del agua de riego.

La recolección del humus y la lombriz se realiza cada dos o tres meses cuando se duplique la cantidad de lombrices evitando tener sobrepoblación, puesto que esta dificultaría las funciones fisiológicas por los espacios reducidos; se aconseja mantener una población baja de 2500 a 3000 individuos $/ \mathrm{m}^{2}$. Las lombrices deben ser llevadas a estrés alimenticio para poder hacer la cosecha, además de esto se deberá realizar posteriormente análisis de la calidad fitosanitaria y nutricional. (García, 2005)

\section{Reciclaje de basura}

Dada la poca cantidad de cultivos en cercanías de la ciudad de Bogotá que emplean abonos orgánicos, y que basan su actividad productiva en el uso de abonos de tipo químico, que además de tener un elevado costo, deterioran la calidad del suelo. La propuesta sugiere un tipo de desecho especial, en primera medida por el contexto de la propuesta y por otro lado dada la ausencia de abundantes desechos animales que faciliten la producción de abono orgánico, se tomara basura producida por la ciudad. (Carvajal, 1996).

Conforme a las necesidades ya presentadas y aspectos de tipo social, se considera importante que la comunidad universitaria conozca la actividad del reciclaje y en especial del compostaje, para generar desde sus actividades diarias, el correcto uso y manejo de sus residuos a favor de la sustentabilidad.

\section{Sustentabilidad}

Imaginemos una partícula del residuo a tratar formada por: materia orgánica de fácil degradación, una fracción de materia orgánica con una resistencia parcial a ser degradada biológicamente y una fracción mineral que resultará inalterada por el proceso. La fracción de materia orgánica fácilmente degradable está compuesta principalmente por hidratos de carbono de cadena corta, fundamentalmente monosacáridos y oligosacáridos, lípidos sencillos, proteínas y algunos tipos de 
Bio-grafía Escritos sobre la Biologia y su Enseñanza.

Edición Extra-Ordinaria. ISSN 2027-1034 P. p113 - 121

Memorias del VII Encuentro Nacional de Experiencias en la Enseñanza de la

Biología y la Educación Ambiental y 11 Congreso Nacional de Investigación en la

Enseñanza de la Biología

polisacáridos como la hemicelulosa. La fracción más resistente a la degradación variará según el origen de la materia orgánica, pero básicamente la componen celulosas y ligninas junto con lípidos complejos. Los microorganismos presentes en el material residual inician su actividad de degradación superficial sobre la materia orgánica mediante la liberación de enzimas extracelulares, para lo que es preciso una cierta humedad en la superficie del material. La micro-fauna aerobia tiene una tasa de degradación mayor, por lo que según aumente su actividad se incrementará el consumo de oxígeno, pudiendo llegar a crearse un medio anaerobio si las condiciones físicas del material no son las adecuadas o no hay un control del proceso.

Si las condiciones son adecuadas la fracción de materia orgánica fácilmente degradable será la primera en ser atacada por los microorganismos, obteniéndose como metabolitos finales de su descomposición $\mathrm{CO}_{2}, \mathrm{H}_{2} \mathrm{O}$ y muy frecuentemente $\mathrm{NH}_{3}$, que se liberarán principalmente en forma gaseosa. Esta actividad metabólica provoca un rápido auto-calentamiento de la masa por la liberación en forma de calor de la energía contenida en los enlaces químicos de las moléculas degradadas. Este incremento de la temperatura será el que marque las diferentes fases del proceso, favoreciendo a distintos conjuntos de microbiota e inhibiendo a otros según los valores que se van alcanzando. En cuanto a la fracción de materia orgánica más resistente, su degradación en los primeros estados del proceso no será más que parcial, aunque contribuirá a la generación de calor y se dará un desprendimiento de $\mathrm{CO}_{2}$ y $\mathrm{H}_{2} \mathrm{O}$ principalmente, siendo muy baja si la hay, la producción de $\mathrm{NH}_{3}$. (Rodríguez, 2000).

Todo lo anteriormente enunciado articula de manera directa las temáticas que se trataran en el aula de clase, y que facilitaran la enseñanza de la temáticas propias de cada asignatura, de esta manera refiriéndose a las nuevas enseñanzas y a las nuevas experiencias prácticas se espera mejorar la sustentabilidad de la comunidad educativa. Hablar de sustentabilidad en el aula implicaría todo una controversia, de hecho no está lejano de ser considerado como tal, asumiendo la sustentabilidad como la "visión de futuro que tome en cuenta las expectativas y necesidades que tendrán las generaciones venideras" (Briceño, 2003), se difiere básicamente por el proceso que implica manejar estas temáticas en el aula de clase tratando de profesores en formación, puesto que su profesión implica la interdisciplinariedad manejada desde diferentes contextos como la educación ambiental, en esta medida se espera desarrollar los análisis químicos que reflejen los beneficios del proceso y por otro lado reconocer la implicaciones científicas, sociales, éticas y ambientales del mismo. El concepto de desarrollo sustentable se hizo conocido mundialmente a partir del informe "Nuestro Futuro Común", publicado en 1987 con motivo de la preparación para la Conferencia Mundial de 
Bio-grafía Escritos sobre la Biologia y su Enseñanza.

Edición Extra-Ordinaria. ISSN 2027-1034 P. p113-121

Memorias del VII Encuentro Nacional de Experiencias en la Enseñanza de la

Biología y la Educación Ambiental y II Congreso Nacional de Investigación en la Enseñanza de la Biología

las Naciones Unidas sobre Medio Ambiente y Desarrollo, realizada en Río de Janeiro, Brasil, en 1992.

Actualmente a nivel mundial se trabaja desde diferentes áreas sobre herramientas y procesos que mitiguen en alguna medida el deterioro ambiental al cual se está enfrentando el planeta, dando cabida a nuevos conceptos como la agroecología, en este contexto y refiriéndose puntualmente al desarrollo de la agricultura en busca de una estable calidad ambiental, que más allá de lograr beneficiar al sistema productivo busca alcanzar: "la seguridad alimentaria, erradicar la pobreza y conservar y proteger el medio ambiente y los recursos naturales" (Altieri y Nicholls, 2000). Por lo anterior se relacionan los objetivos más comunes de las definiciones de agricultura sustentable (Altieri y Nicholls, 2000): - Producción estable y eficiente de recursos productivos. - Alto nivel de participación de la comunidad - Conservación y generación de recursos naturales -Autosuficiencia alimentaria.

La meta de la sustentabilidad es el esfuerzo conservativo para mantener el sentido tradicional y los niveles de ingreso en una era en la cual el capital natural no es ya un bien ilimitado, al contrario más y más un factor limitante del desarrollo (Campus de Educación Educativa, 2010); debe convertirse en un paradigma alternativo en el cual los recursos ambientales, como potenciales capaces de reconstruir el proceso económico dentro de una nueva racionalidad productiva, promuevan un proyecto social fundado en las autonomías culturales, en la democracia y en la productividad de la naturaleza (CEE, 2010).

\section{Educación Ambiental}

Cuando se aborda el campo de la educación ambiental, se puede considerar que a pesar de su preocupación común por el medio ambiente y el reconocimiento del papel central de la educación para el mejoramiento de la relación con este, los diferentes autores (investigadores, profesores, pedagogos, animadores, asociaciones, organismos, etc.) adoptan diferentes discursos sobre la EA y proponen diversas maneras de concebir y de practicar la acción educativa en este campo. Cada uno muestra su propia visión y se ha incluso visto erigirse «capillas» pedagógicas que proponen la manera «correcta» de educar, «el mejor» programa, «el» método adecuado. Por lo cual Una de las estrategias de aprehensión de las diversas posibilidades teóricas y prácticas en el campo de la educación ambiental consiste en elaborar un mapa de este «territorio» pedagógico. Se trata de reagrupar proposiciones semejantes en categorías, de caracterizar cada una de estas últimas y de distinguirlas entre ellas, poniéndolas al mismo tiempo en relación divergencias, puntos comunes, oposición y complementaria. (Sauvé, 2010) 
Memorias del VII Encuentro Nacional de Experiencias en la Enseñanza de la

Biología y la Educación Ambiental y 11 Congreso Nacional de Investigación en la Enseñanza de la Biología

\section{Metodología}

El mecanismo de implementación de esta propuesta consistirá en tres etapas; en las cuales lo primero que se dispondrá a realizar es una inducción de la técnica del compostaje a la comunidad educativa, se continua con el proceso de enseñanza de reciclaje de residuos aprovechables y finalmente se ejecuta el tratamiento del compost dado desde los residuos orgánicos para obtener el Humus; se establecerá notificaciones murales y el uso de las TIC's será indispensable para la distribución de la información.

Durante la segunda etapa de la propuesta se determinara los antecedentes y posibles evaluaciones realizadas en el área del Departamento de Química en cuanto al manejo de los residuos que se presentan diariamente por parte de la comunidad, con ello se hará un diagnostico primario de mejoramiento de las posibles estrategias que se pueden establecer para la mitigación, control y disposición final de residuos ordinarios del entorno.

Por último y luego de haber realizado las actividades presentadas en las tres etapas, se realiza una evaluación del proceso que pueda presentar un diagnóstico de la eficacia del compostaje y el reciclaje en el Departamento de Química. (González, Sánchez y Areal, 2013).

Para llevar a cabo el proyecto se desarrolla seis actividades las cuales están divididas de la siguiente manera:

Tabla N¹. Metodología de trabajo de actividades de la propuesta establecida. ${ }^{4}$

\begin{tabular}{|c|l|l|l|}
\hline ACTIVIDAD & \multicolumn{1}{|c|}{ FORMULACION } & \multicolumn{2}{|c|}{ MECANISMO } \\
\hline & Conocer e identificar los posibles & Se emplea una matriz \\
CARACTERIZA & residuos que se puedan aprovechar & pre- proceso de impacto \\
en el departamento de química de la & ambiental basada en la \\
CIÓN DE LOS & Universidad Pedagógica Nacional, al & observación, normatividad \\
RESIDUOS & igual que conocer los antecedentes & vigente y en las últimas \\
& por los cuales se establece una & visitas realizadas por el \\
ente controlador.
\end{tabular}

${ }^{4}$ Las actividades están dadas para un tiempo de 6 semanas, lo cual cada actividad dispondrá de una semana para su ejecución. 
Bio-grafia Escritos sobre la Biología y su Enseñanza.

Edición Extra-Ordinaria. ISSN 2027-1034 P. p113 - 121

Memorias del VII Encuentro Nacional de Experiencias en la Enseñanza de la Biología y la Educación Ambiental y II Congreso Nacional de Investigación en la

Enseñanza de la Biología

\begin{tabular}{|c|c|c|}
\hline & $\begin{array}{l}\text { comunidad estará en mayor contacto } \\
\text { con la ejecución del proyecto. }\end{array}$ & $\begin{array}{lll}\text { sostenibilidad } & \text { del } \\
\text { compostaje para } & \text { así } \\
\text { conocer la aplicación. } & \\
\end{array}$ \\
\hline $\begin{array}{l}\text { EJECUCIÓN } \\
\text { DEL } \\
\text { PROCESO DE } \\
\text { COMPOSTAJE }\end{array}$ & $\begin{array}{l}\text { Para la aplicación del compostaje se } \\
\text { emplea la lombriz roja californiana, y } \\
\text { se realiza el proceso de separación lo } \\
\text { que permitirá distinguir los residuos } \\
\text { biodegradables que se emplean para } \\
\text { dicho compost. Esto se realizara a } \\
\text { las condiciones óptimas que la } \\
\text { fundamentación teórica nos brinda. }\end{array}$ & $\begin{array}{l}\text { El alimento de las } \\
\text { lombrices se da por medio } \\
\text { de la recolección realizada } \\
\text { por las personas } \\
\text { vinculadas a los espacios } \\
\text { académicos inmersos en } \\
\text { este proyecto, con las } \\
\text { cuales se aplica lo tratado } \\
\text { en la inducción sobre el } \\
\text { tratamiento de la técnica }\end{array}$ \\
\hline $\begin{array}{l}\text { RECOLECCIO } \\
\text { N DEL } \\
\text { COMPOSTAJE }\end{array}$ & $\begin{array}{l}\text { Cuando se tenga una estimación de } \\
20 \text { a } 30 \text { días después de la primera } \\
\text { reproducción de las lombrices se } \\
\text { hará el conteo. }\end{array}$ & $\begin{array}{l}\text { Recolección del humus, el } \\
\text { cual se pesa y controla } \\
\text { mensualmente. }\end{array}$ \\
\hline $\begin{array}{l}\text { DISPOSICIÓN } \\
\text { FINAL DE LOS } \\
\text { DEMÁS } \\
\text { RESIDUOS }\end{array}$ & $\begin{array}{l}\text { Es claro que no se pueden obviar los } \\
\text { demás residuos que se separaron en } \\
\text { la primera etapa de las actividades. }\end{array}$ & $\begin{array}{l}\text { Se realiza campañas } \\
\text { mensuales de recolección } \\
\text { de residuos reutilizables } \\
\text { como el cartón, papel, etc., } \\
\text { y se hará una } \\
\text { reintegración al ciclo } \\
\text { productivo. }\end{array}$ \\
\hline $\begin{array}{c}\text { ANÁLISIS Y } \\
\text { CUANTIFICACI } \\
\text { ÓN DEL } \\
\text { MEJORAMIEN } \\
\text { TO }\end{array}$ & $\begin{array}{l}\text { Conforme al tiempo estimado para } \\
\text { validar los resultados obtenidos por la } \\
\text { aplicación de dicho proceso de } \\
\text { compost. }\end{array}$ & $\begin{array}{l}\text { Se dispondrá nuevamente } \\
\text { a validación una matriz de } \\
\text { impacto para identificar el } \\
\text { funcionamiento } \\
\text { proyecto. }\end{array}$ \\
\hline
\end{tabular}

\section{Consideraciones Finales}

La propuesta encamina su expectativa de manera lejana a la construcción de un sistema basado en la lombricultura, lo que se espera y lo que se plantea como meta, es lograr una aproximación a la transformación del pensamiento y cultura entorno al manejo de residuos; apoyado desde la lombricultura. Claramente en la actual sociedad de consumo, la cantidad exagerada de producción de residuos requiere un manejo adecuado y provecho de estos, lograr hacerlo solo se alcanzaría si antes se genera el impacto y aceptación por parte de la comunidad, la Educación verde propende un papel integrador desde el aspecto social, pero su razón de ser nace desde la necesidad de educar a la población de manera 
Memorias del VII Encuentro Nacional de Experiencias en la Enseñanza de la Biología y la Educación Ambiental y 11 Congreso Nacional de Investigación en la

\section{Enseñanza de la Biología}

sustentable para el ambiente, por eso la enseñanza desde estrategias vinculadas a medios de producción de bajo costo y de carácter conservacionista resultan de gran ayuda para generar el cambio en las percepciones. La enseñanza de este aspecto ambiental resulta cumbre en la formación inicial de profesores de ciencias debido a su formación disciplinar, la cual implica un continuo contacto con estas problemáticas que posteriormente se verán reflejadas en los salones de clase de los colegios, por ende es indispensable en la interdisciplinariedad de los docentes en formación el manejo de esta temática, para que logren tratarlas correctamente y generen la cultura del correcto manejo de residuos sólidos en sus futuros estudiantes.

\section{Resultados y Discusión}

Los resultados logrados con la estrategia de enseñanza, esperan ser sujetos de un análisis detallado, a partir de cada dimensión abordada desde la sustentabilidad, claramente los resultados de esta primera etapa consiste en la implementación de la metodología estratégica. El proceso de enseñanza desarrollado espera alcanzar los fines fijados en cuanto a la mitigación de los residuos pero en mayor grado de la cultura ambiental lograda a través de la enseñanza.

\section{Bibliografía}

Altieri, M. y Nicholls, C. (2000). Agroecología: Teoría y práctica para una agricultura sustentable ( $1^{\circ}$ edición). Programa de las Naciones Unidas para el Medio Ambiente.

Barbado, J. (2004). Cría de lombrices. Buenos Aires: Albatros, 128 p.

Briceño, M. (2003). Universidad, Sector productivo y sustentabilidad. Universidad Central de Venezuela.

Campus de Educación Ejecutiva. (2010). El concepto de sustentabilidad y la importancia de cuidar el Medio Ambiente. Extraído el 14/10/2013 de: extensionacademica.wordpress.com/2010/03/26/el-concepto-de-sustentabi lidad-yla-importancia-de-cuidar-el-medio-ambiente

Carvajal, J. (1996). Manual de prácticas agroecológicas de los Andes Ecuatorianos. Editorial Abya Yala.

García, M. (2005). Manual de cría de la lombriz de tierra: una alternativa ecológica y rentable. Bogotá: Editorial taller de San Pablo. 
Bio-grafía Escritos sobre la Biología y su Enseñanza.

Edición Extra-Ordinaria. ISSN 2027-1034 P. p113 - 121

Memorias del VII Encuentro Nacional de Experiencias en la Enseñanza de la Biología y la Educación Ambiental y 11 Congreso Nacional de Investigación en la Enseñanza de la Biología

Gonzalez, M., Sanchez, M. y Areal, C. Unidad Didáctica: Elaboración de Compost. Extraído el 15 de Julio de 2013 de: http://webs.uvigo.es/msuare zMEB_CAP_Unidade6.pdf.

Padilla, F. y Cuesta, A. (2003). Zoología Aplicada. España: Ediciones Díaz de Santos, S. A.

Rodríguez, A. (2000). Marco Normativo para el uso del Compost. Madrid: MIMAM.

Rodríguez, F. (2005). Lombricultura para pequeños emprendedores. Manual teórico-práctico para el manejo comercial de la lombriz roja californiana. Editorial La Quimera.

Sauvé, L. (2010). Educación científica y educación ambiental: un cruce fecundo. Revista Enseñanza de las Ciencias. N²8. p. 5-18.

Schuldt, M. (2006). Lombricultura: Teoría y Práctica. Madrid: Mundi-prensa. 